\section{Uniform Inhibition of Dopamine Neurons in the Ventral Tegmental Area by Aversive Stimuli}

\author{
Mark A. Ungless, ${ }^{*} \dagger$ Peter J. Magill, J. Paul Bolam
}

\begin{abstract}
Dopamine neurons play a key role in reward-related behaviors. Reward coding theories predict that dopamine neurons will be inhibited by or will not respond to aversive stimuli. Paradoxically, between 3 and $49 \%$ of presumed dopamine neurons are excited by aversive stimuli. We found that, in the ventral tegmental area of anesthetized rats, the population of presumed dopamine neurons that are excited by aversive stimuli is actually not dopaminergic. The identified dopamine neurons were inhibited by the aversive stimulus. These findings suggest that dopamine neurons are specifically excited by reward and that a population of nondopamine neurons is excited by aversive stimuli.
\end{abstract}

Dopamine neurons of the ventral tegmental area (VTA) and substantia nigra pars compacta exhibit rapid and brief bursts of activity in response to unexpected rewarding stimuli or conditioned stimuli associated with those rewards. When a reward becomes expected, dopamine neurons no longer fire; if the reward fails to occur, dopamine neurons are inhibited (1). It has thus been suggested that dopamine neurons encode a reward error-prediction rule (1). In addition, dopamine neurons might encode incentive salience for rewards $(2,3)$. The reward aspect of these hypotheses predicts that aversive stimuli should inhibit (or at least not excite) dopamine neurons. Most studies find that about $80 \%$ of presumed dopamine neurons are inhibited by or do not respond to aversive stimuli. However, between 3 and $49 \%$ of presumed dopamine neurons are excited by aversive stimuli (49 ). This finding clearly represents a major challenge to the reward hypotheses. In these studies, dopamine neurons have typically been identified as dopaminergic on the basis of their electrophysiological properties alone (10). Combined in vivo physiology and labeling experiments have confirmed that dopamine neurons do indeed has been found in vitro that a population of nondopaminergic neurons exists in the VTA with similar electrophysiological characteristics to dopamine neurons but with slightly narrower action potentials (12). Presumed dopamine neurons that are excited by aversive stimuli often display a

Medical Research Council Anatomical Neuropharmacology Unit, Department of Pharmacology, University of Oxford, Mansfield Road, Oxford OX1 3TH, UK.

*Present address: Department of Zoology, University †To whom correspondence should be addressed. Email: mark.ungless@zoo.ox.ac.uk possess these properties (11). However, it of Oxford, South Parks Road, Oxford OX1 3PS, UK. narrower action potential than those neurons that are inhibited (7). We therefore directly tested the possibility that the presumed dopamine neurons that are excited by aversive stimuli are not actually dopaminergic, and that, consistent with the reward hypothesis of their function, dopamine neurons are uniformly inhibited by aversive stimuli.

We recorded extracellular unit activity from single VTA neurons in anesthetized rats (13). Using standard electrophysiological criteria, we identified presumed dopamine neurons (10). We observed both excitatory and inhibitory responses to a standard aversive stimulus (foot pinch) (14). Neurons were subsequently labeled with the use of the juxtacellular technique (15) and neurochemically characterized with immunofluorescence for tyrosine hydroxylase (TH), the essential ratelimiting enzyme for dopamine synthesis. Neurons that were immunopositive for TH, and therefore dopamine releasing $(n=12$; Fig. 1, A and B), were typically inhibited by the aversive stimulus [10 of 12; Fig. 1C; mean change $( \pm \mathrm{SEM})=-0.82 \pm 0.23 \mathrm{~Hz}$, $P<0.005]$. In contrast, neurons that were immunonegative for TH (i.e., nondopamine; $n=6$; Fig. 1, D and E) were typically excited by the aversive stimulus ( 4 of 6 ; Fig. 1F). These TH-negative neurons often exhibited burst firing (Fig. 1E) and an initial segment spike in the action potential, both of which are commonly considered to be characteristic of dopamine neurons (11). They were always in close proximity to TH-positive somata and within the dense TH-positive processes of the VTA (Fig. 1D).

Although the identified dopamine neurons had higher firing rates than the nondopamine neurons, the range of dopamine neuron firing rates fell entirely within the range of nondopamine neuron firing rates (dopamine: $4.6 \pm$ $0.2 \mathrm{~Hz}$, range 1.6 to $5.9, n=12$; nondopamine: $2.6 \pm 1.1 \mathrm{~Hz}$, range 0.3 to $7.7, n=6$; $P<0.05$ ), and both groups had similar action potential amplitudes (dopamine: $0.75 \pm 0.1$ $\mathrm{mV}$, range 0.32 to 1.32 ; nondopamine: $1.3 \pm$ $0.4 \mathrm{mV}$, range 0.35 to $3.28 ; P>0.05$ ). All dopamine and nondopamine neurons had triphasic action potentials with widths that were greater than $2 \mathrm{~ms}$ but were broader in dopamine neurons than in nondopamine neurons (dopamine: $4.6 \pm 0.2 \mathrm{msec}$, range 4.1 to 5.4; nondopamine: $3.8 \pm 0.4 \mathrm{msec}$; range 2.4 to $4.9 ; P<0.05)$. Because there was considerable overlap of the firing rates and triphasic action potential widths of the two
Fig. 1. Identified dopamine neurons are inhibited by an aversive stimulus; excited neurons are not dopaminergic. (A) A neuron labeled with Neurobiotin (NB) is $\mathrm{TH}$-positive and, therefore, dopaminergic. (B) Average extracellular waveform and baseline firing properties of the labeled dopamine neuron. (C) Firing of the dopamine neuron is decreased during the aversive stimulus. (D and $\mathbf{E}$ ) Examples of baseline firing and average extracellular waveform of a neuron that is $\mathrm{TH}$-negative and, therefore, nondopaminergic. (F) The nondopaminergic neuron was excited by the aversive stimulus. Data in (A) to (C) are from the same TH-positive neuron; data in (D) to $(F)$ are from the same $\mathrm{TH}$-negative neuron. Scale bars in $(A)$ to (C) are the same as those in (D) to (F).

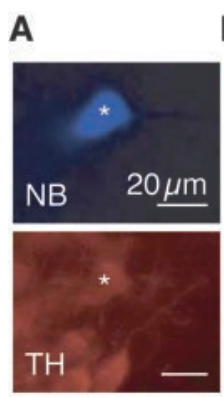

B
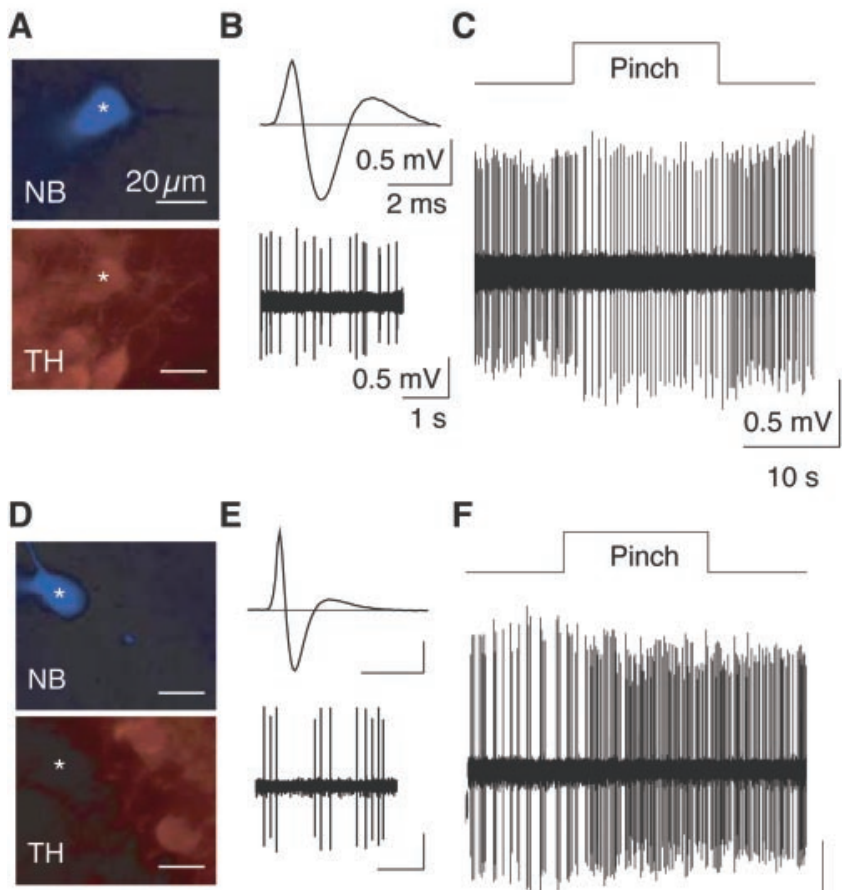

$\mathbf{F}$

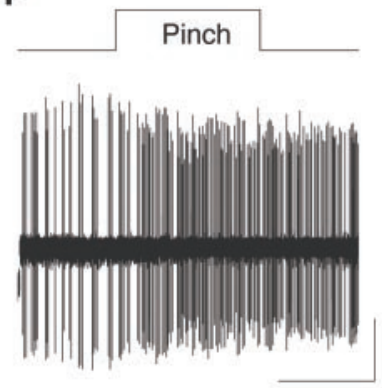


A

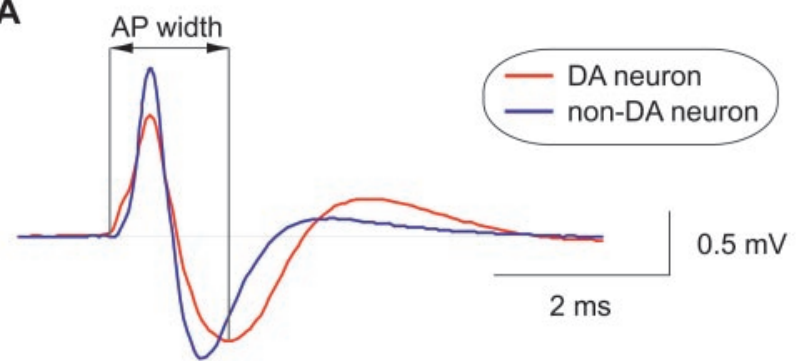

Fig. 2. Proposed criterion for identification of dopamine (DA) neurons. (A) Typical examples of average extracellular waveform of dopaminergic (red) and nondopaminergic (blue) neurons. (B) Neurons that are excited by aversive stimuli have narrower action potentials (AP width). Action potential widths were measured from the start of action potential to the negative trough. (C) A frequency histogram of all recorded neurons shows a biphasic distribution (gray bars). A double-Gaussian fit (blue and red lines) suggests a criterion of $\geq 1.1 \mathrm{msec}$ (from start to negative trough) for the exclusion of nondopamine neurons.

B

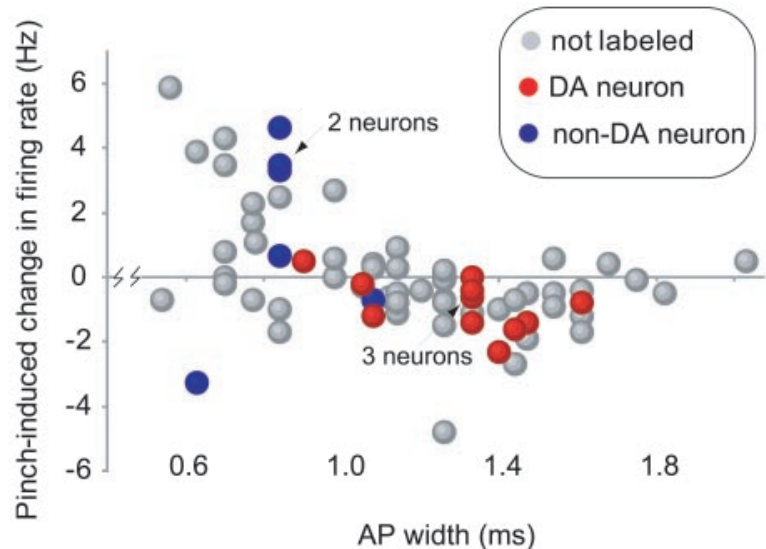

\section{C}

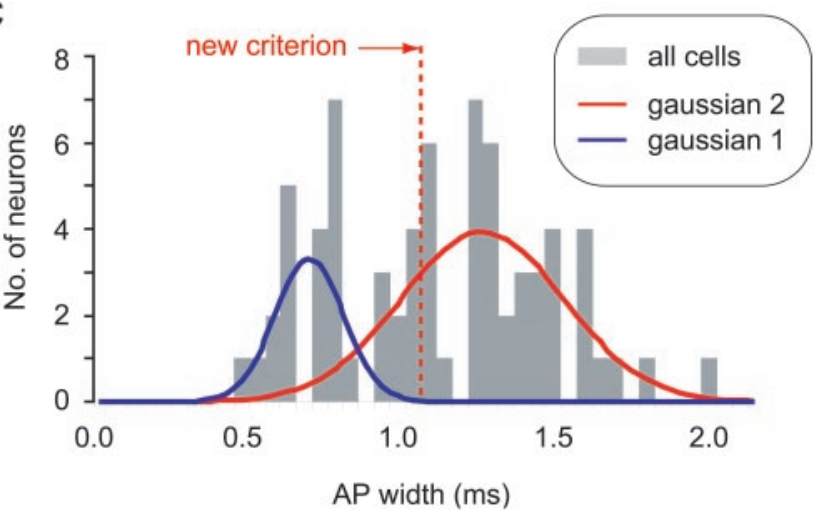

groups, we sought another electrophysiological measure that might allow us to distinguish more clearly between the two groups. The duration from the start of the action potential to the negative trough was significantly broader in dopamine neurons $(P<0.001$; Fig. 2A). An analysis of all of the neurons we recorded (labeled and unlabeled; total $n=70$ ) segregated the population into two groups on the basis of this criterion (Fig. 2B). Both the raw data and a predicted double Gaussian show that a duration of $\geq 1.1 \mathrm{~ms}$ from the start of the action potential to the negative trough should exclude all nondopamine neurons and that, as a result, this may be a useful guide for the future identification of VTA neurons (Fig. 2C).

Because dopamine neurons can regulate their firing pattern and firing rate through independent mechanisms $(16,17)$, we next examined whether aversive stimuli also changed the firing pattern of dopamine neurons. In the absence of salient stimuli, (putative) dopamine neurons typically display slow irregular firing, with intermittent burst firing in vivo. At firing frequencies achieved during bursts $(>12 \mathrm{~Hz})$, dopamine release increases in a supralinear fashion because the dopamine transporter is overwhelmed (18). Therefore, decreased bursting could decrease dopamine release

Fig. 3. Dopamine neurons exhibit reduced burst firing during aversive stimuli. (A) Examples of firing during a baseline period and an aversive stimulus. Firing rate and incidence of bursts were both decreased by the pinch. (B) Dopamine neurons (DA; black) and presumed dopamine neurons ( $\mathrm{PDA}$; gray) exhibit fewer action potential bursts during the aversive stimulus. (C) The probability of action potentials occurring in a burst is decreased during aversive stimuli [DA neurons (black); PDA neurons (gray)]. (D) Changes in firing rate are not correlated with changes in the probability (prob.) that an action potential is in a burst. $*, P<0.05$.

A

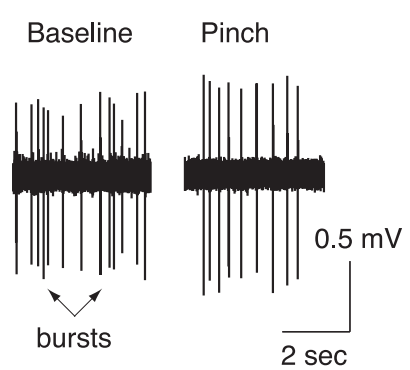

C

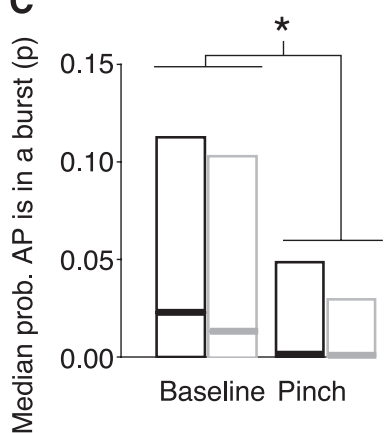

B

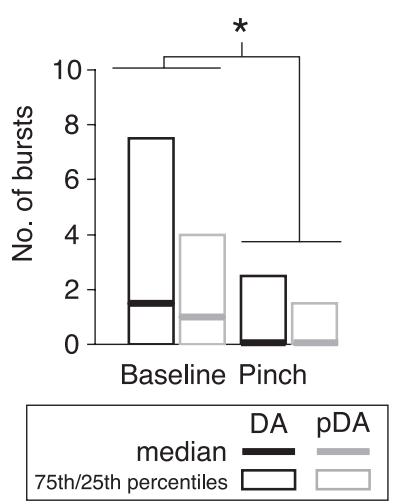

D

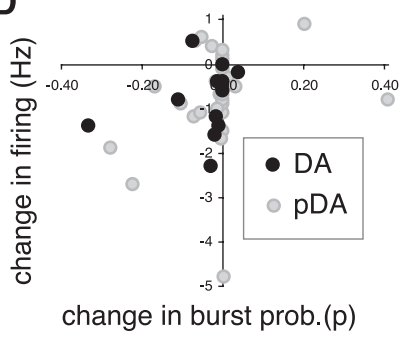

in addition to the effects of a reduction in firing rate. Because many dopamine neurons do not always fire in a bursting mode (19), we increased our sample size by conducting our analysis on identified (i.e., TH- positive) and putative dopamine neurons (identified with our electrophysiological criterion). In these neurons, we observed a reduction in the number of action potentials that occurred in a burst during the pinch 
Fig. 4. Dopamine neurons do not show gradual increases in tonic firing rates after aversive stimuli. (A) Average firing of identified dopamine neurons during first and second pinch (error bars show the mean + SEM). (B) Baseline firing rates of dopamine neurons (DA; black circles) and presumed dopamine neu-

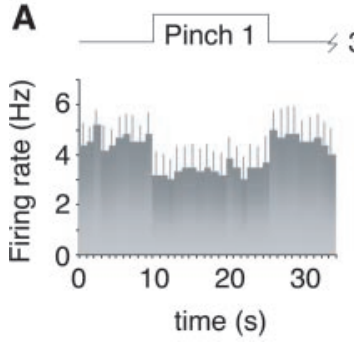

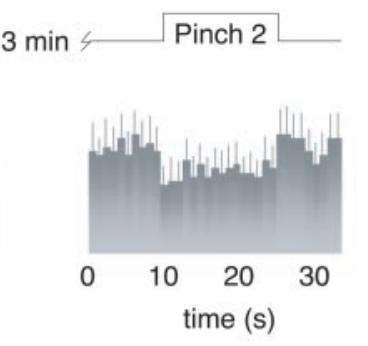

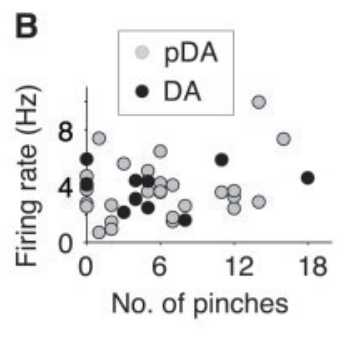

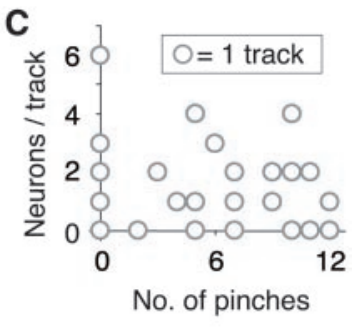

rons (pDA, identified with the proposed criterion; gray circles) are not correlated with the number of aversive stimuli occurring earlier in the experiment. (C) Number of neurons found per track is not correlated with the number of aversive stimuli occurring earlier in the experiment.

(Fig. 3, A and B). The probability that an action potential occurred in a burst was also reduced during the pinch (Fig. 3C), which shows that the reduction in burst number is not simply a result of a reduction in the number of action potentials. We found no correlation between the effects of the aversive stimulus on firing rate and bursts [correlation coefficient $(r)=0.25 ; P>0.05$; Fig. 3D], suggesting that they are regulated through separate mechanisms.

There is considerable evidence that aversive stimuli can slowly increase extrasynaptic dopamine levels over longer time scales (minutes to hours) $(20,21)$. One interesting consequence of this increased tonic extrasynaptic dopamine is the expectation that it will inhibit phasic synaptic dopamine release through presynaptic dopamine D2 receptors $(22,23)$. It has been suggested that this gradual increase in tonic dopamine levels involves changes in the tonic firing rates of the dopamine neurons themselves (24), although it may also involve local actions of other neurotransmitters, such as glutamate, on dopamine release at axon terminals (25). The firing of the identified dopamine neurons returned to baseline levels immediately after the aversive stimulus (Fig. 4A). For cases in which a second pinch was administered $3 \mathrm{~min}$ later, it was clear that firing rates had not slowly increased, and that after this second pinch, firing rates rapidly returned to baseline levels (Fig. 4A). We found no correlation between baseline firing rates of neurons and the number of pinches that occurred earlier in the experiment $(r=0.2$; $P>0.05$; Fig. 4B). We also found no correlation between the number of neurons detected during a track [a commonly used measure of changes in dopamine neuron activity (16)] and the previous number of pinches $(r=-0.027 ; P>0.05$; Fig. $4 \mathrm{C})$.

These results show that VTA neurons that are excited by aversive stimuli are not dopaminergic. Dopamine neurons were uniformly inhibited by aversive stimuli. These data further demonstrate the presence of a functionally distinct nondopaminergic population of neurons in the VTA, which may have been included in the analysis of dopamine neurons in previous studies that used the classical identification criteria. The refined electrophysiological criterion we propose for distinguishing between these populations of neurons should help future investigations and should also allow for reexamination of data. Although the neurochemical identity of these nondopamine neurons is currently unknown, it is unlikely that they are $\gamma$-aminobutyric acid (GABA)-ergic, because GABAergic neurons in the VTA have markedly different electrophysiological characteristics [e.g., very rapid action potentials $(<1.5 \mathrm{~ms})$ and high firing rates $(>10 \mathrm{~Hz})](26)$.

Although it is not possible to use the juxtacellular labeling technique in freely moving unanesthetized animals, and it may not be practical in primates, it is likely that our findings can be extrapolated to other species and freely moving states for several reasons. First, the basic properties of dopamine neurons in anesthetized and unanesthetized states appear to be very similar, and these properties do not substantially vary across several species $(4,5,27,28)$. Second, stimulus-evoked responses in dopamine neurons, particularly to aversive stimuli, are similar across these different states and species (4-9).

Our results show that aversive stimuli consistently reduce the phasic dopamine signal through a reduction in firing rate and bursting activity, and therefore strengthen the reward hypothesis of dopamine neuron function.

\section{References and Notes}

1. W. Schultz, Neuron 36, 241 (2002).

2. T. E. Robinson, K. C. Berridge, Brain Res. Brain Res. Rev. 18, 247 (1993).

3. S. M. McClure, N. D. Daw, P. R. Montague, Trends Neurosci. 26, 423 (2003).
4. J. Mirenowicz, W. Schultz, Nature 379, 449 (1996).

5. W. Schultz, R. Romo, J. Neurophysiol. 57, 201 (1987).

6. J. Mantz, A. M. Thierry, J. Glowinski, Brain Res. 476, 377 (1989)

7. L. A. Chiodo, S. M. Antelman, A. R. Caggiula, C. G. Lineberry, Brain Res. 189, 544 (1980).

8. H. Maeda, G. J. Mogenson, Brain Res. Bull. 8, 7 (1982).

9. F. A. Guarraci, B. S. Kapp, Behav. Brain Res. 99, 169 (1999).

10. Properties used to identify dopamine neurons include a broad triphasic extracellular action potential of a width greater than $2 \mathrm{~ms}$ and a relatively slow firing rate $(<10 \mathrm{~Hz})$.

11. A. A. Grace, B. S. Bunney, Neuroscience 10, 301 (1983).

12. D. L. Cameron, M. W. Wessendorf, J. T. Williams, Neuroscience 77, 155 (1997).

13. Materials and methods are available as supporting material on Science Online.

14. It is difficult to classify stimuli as aversive in the anesthetized preparation, because no behavioral measurements can be made. However, the foot pinch that we used would be aversive in the unanesthetized state, and because the responses of dopamine neurons to aversive stimuli are not strongly dependent on anesthesia, we believe that such a classification in this case is valid.

15. D. Pinault, J. Neurosci. Methods 65, 113 (1996).

16. S. B. Floresco, A. R. West, B. Ash, H. Moore, A. A. Grace, Nature Neurosci. 6, 968 (2003).

17. P. G. Overton, D. Clark, Brain Res. Brain Res. Rev. 25, 312 (1997).

18. F. G. Gonon, Neuroscience 24, 19 (1988).

19. A. A. Grace, B. S. Bunney, J. Neurosci. 4, 2877 (1984).

20. J. C. Horvitz, Neuroscience 96, 651 (2000).

21. J. D. Salamone, Behav. Brain Res. 61, 117 (1994).

22. A. A. Grace, Neuroscience 41, 1 (1991).

23. P. Palij et al., Brain Res. 509, 172 (1990).

24. K. A. Keefe, A. F. Sved, M. J. Zigmond, E. D. Abercrombie, J. Neurochem. 61, 1943 (1993).

25. H. P. Jedema, B. Moghaddam, J. Neurochem. 63, 785 (1994).

26. S. C. Steffensen, A. L. Svingos, V. M. Pickel, S. J. Henriksen, J. Neurosci. 18, 8003 (1998).

27. M. Fa et al., Synapse 48, 1 (2003).

28. A. S. Freeman, B. S. Bunney, Brain Res. 405, 46 (1987).

29. We thank J. Boyes, T. Klausberger, and J. Tepper for comments on this manuscript; M. Melis, B. Schilstrom, and E. Abercrombie for helpful discussions; and A. Baude, P. Cobden, L. Norman, C. Francis, and B. Micklem for technical assistance. Supported by the Medical Research Council of the UK (J.P.B) and the E. P. Abraham Fund (A. D. Smith). P.J.M. holds a Fellowship by Examination at Magdalen College, Oxford.

Supporting Online Material

www.sciencemag.org/cgi/content/full/303/5666/2040/ DC1

Materials and Methods

References

5 November 2003; accepted 9 February 2004 